\title{
New Conditions for the Exponential Stability of Nonlinear Differential Equations
}

\author{
Rigoberto Medina \\ Departamento de Ciencias Exactas, Universidad de Los Lagos, Casilla 933, Osorno, Chile \\ Correspondence should be addressed to Rigoberto Medina; rmedina@ulagos.cl
}

Received 23 January 2017; Accepted 23 March 2017; Published 10 April 2017

Academic Editor: Daoyi Xu

Copyright (c) 2017 Rigoberto Medina. This is an open access article distributed under the Creative Commons Attribution License, which permits unrestricted use, distribution, and reproduction in any medium, provided the original work is properly cited.

\begin{abstract}
We develop a method for proving local exponential stability of nonlinear nonautonomous differential equations as well as pseudolinear differential systems. The logarithmic norm technique combined with the "freezing" method is used to study stability of differential systems with slowly varying coefficients and nonlinear perturbations. Testable conditions for local exponential stability of pseudo-linear differential systems are given. Besides, we establish the robustness of the exponential stability in finite-dimensional spaces, in the sense that the exponential stability for a given linear equation persists under sufficiently small perturbations. We illustrate the application of this test to linear approximations of the differential systems under consideration.
\end{abstract}

\section{Introduction}

The stability and robustness of differential systems have been widely investigated over the past decades; see, for example, [1-7] and references therein. This is due to theoretical interests and to being a powerful tool for system analysis and control design. The stability and robustness are the basic requirements for controlled systems. In practice, to satisfy the performance specification and to have a good transient response of the system, the controlled system is often designed to possess a stability degree. If the controlled system has a stability degree $\alpha$, we say that the system is exponentially stable. The concept of $\alpha$-stability is related to the exponential stability with a convergence rate $\alpha>0$.

Unlike the situation for linear systems, where necessary and sufficient conditions for stability are provided, the nonlinear problem is not completely solved. In fact, in spite of recent efforts (see [8-13] and the references therein), the exponential stability problem of nonlinear nonautonomous systems can be considered largely open. The main technique to stability of differential systems is Lyapunov's method and its variants (Razumikhin-type theorems, LyapunovKrasovskii functional techniques); see, for example, [10, 1416]. In contrast, many alternative methods to Lyapunov's functions have been successfully applied to the stability analysis of differential systems, for example, Ngoc [11], assuming that a nonlinear differential system with time-varying delay is bounded above by a positive linear time-invariant differential system and if this last system is exponentially stable then the nonlinear system under consideration is also exponentially stable. Anderson et al. [17], using the concept of Lyapunov exponents and Bohl exponents, discuss the problem of stabilization for linear time-varying systems with bounded matrices. Coppel [15], using the concept of ordinary and exponential dichotomy, establishes new results in stability theory, and also the "freezing" method became a fruitful tool among those alternative approaches; see, for example, Vinograd [18] and Gil and Medina [19]. In particular, the latter has been applied to prove that exponential stability of linear time-invariant differential systems implies the exponential stability of the system under consideration, provided that the coefficients of the original differential system are slowly varying. Moreover, an important tool to obtain explicit stability criteria for linear differential systems is the logarithmic norm of matrices (measure of matrices), which were used effectively in the recent literature on investigations of equations with dissipative coefficient matrices and their perturbations; see, for example, Zevin and Pinsky [12]. Besides, the logarithmic norm has been used to study the error bounds in the numerical integration of 
ordinary differential equations [20,21], estimates or stability of differential equations [15], and the oscillatory behavior of retarded functional differential equations [22].

Pseudo-linear systems are an important class of nonlinear systems. The stability and robustness of pseudo-linear differential equations are considered, for example, in [8, 10, 23-25].

Banks et al. [8] and Martynyuk [25] derived new bounds for solutions of perturbed pseudo-linear differential equations, basically using Gronwall-type inequalities. Dvirnyi and Slyn'ko $[23,24]$, constructing a piecewise differential Lyapunov function, established the stability of solutions to impulsive differential equations with impulsive action in the pseudo-linear form. Banks et al. [8], using a Gronwall-type inequality and assuming that a matrix $B(x, t)$ satisfies a jointly Lipchitz inequality in $x$ and $t$, established the robust exponential stability of evolution differential equations of pseudolinear form. In summary, in the existing literature there are many results concerning the stability or asymptotic behavior of pseudo-linear differential equations; however, in general, the assumptions are difficult to check or conservative.

The purpose of this paper is to establish explicit conditions for the exponential stability of nonlinear differential systems. This approach led to study special classes of control systems, for example, systems with linear compact operators. In fact, assuming appropriate conditions on the perturbation term, the exponential feedback stabilization of a class of time-varying nonlinear systems can be established, provided the rate of variation of the system coefficients operators is sufficiently small.

In this paper we consider differential systems defined in Euclidean spaces, with bounded operators on the right-hand side represented in the pseudo-linear form. New estimates for the norms of solutions are derived giving us explicit stability and boundedness conditions. The equations will be represented as a perturbation about a fixed value of the coefficient operator. Thus, applying norm estimates for the involved operator-valued functions, new stability results are established.

The structure of this paper is as follows: in Section 2, we introduce some notations, the concept of stability with respect to a ball, and the definition and its properties of the logarithmic norm functions. In Section 3, the main exponential stability results and its consequences are established for nonlinear differential equations. In Section 4, we extend the main results to pseudo-linear differential systems. In Section 5, we applied the results of Section 4 to a linear approximation of the considered nonlinear system. Finally, Section 6 is devoted to the discussions of our results: in fact, the results are interpreted appropriately and robust conclusions are drawn.

\section{Preliminaries}

Let $C\left([a, b], R^{n}\right)$ be the set of all $R^{n}$-valued continuous functions on $[a, b]$.

Let us consider a system described by the following equation in the Euclidean space $R^{n}$ :

$$
\frac{d x}{d t}=A(t) x(t)+F(t, x(t)), \quad t \geq 0,
$$

where $A(t)$ is a matrix-valued function, continuous and uniformly bounded on $[0, \infty)$, and $F:[0, \infty) \times R^{n} \rightarrow R^{n}$ is a nonlinear and continuous vector function. The existence of solutions is assumed.

For a number $\eta \in(0, \infty]$, put

$$
\Omega(\eta)=\left\{h \in R^{n}:\|h\| \leq \eta\right\} .
$$

We will use $\|\cdot\|$ to denote norms in $R^{n}$ and $R^{n \times n}$, respectively.

Definition 1. The zero solution of system (1) is exponentially stable with respect to a ball $\Omega(\lambda)$ if there are constants $\beta, M>0$, such that for any solution $x\left(t, t_{0}\right)$ of $(1)$, with initial condition $x\left(t_{0}, t_{0}\right)=x_{0} \in \Omega(\lambda)$, the following inequality holds:

$$
\left\|x\left(t, t_{0}\right)\right\| \leq M e^{-\beta\left(t-t_{0}\right)}\left\|x_{0}\right\|, \quad \forall t \geq t_{0} \geq 0 .
$$

Remarks 1. (1) The stability analysis with respect to a ball has been considered by many researchers (see, e.g., Furuta and Kim [26] and Hsiao et al. [27]). However, this kind of stability is defined in terms of the set of roots of characteristic polynomials corresponding to linear autonomous delay equations. In contrast, the concept of stability with respect to a ball used in this paper allows us to characterize the region of attraction of exponential stability of nonautonomous differential equations. Furthermore, in our case, the radius of the ball $\Omega(\lambda)$ can be explicitly calculated in terms of known quantities.

(2) We want to point out that, considering solutions with initial functions into the region $\Omega(\lambda)$, we will ensure reasonable dynamics, for example, exponential decay rates.

Definition 2. System (1) is said to have a stability degree $\alpha$ (or to be exponentially stable), with $\alpha>0$, if $z(t)=[\exp (\alpha t)] x(t)$ is a bounded function, with $x(t)$ a solution of (1). In this case, the parameter $\alpha$ is called the convergence rate.

It is assumed that, for a positive number $r \leq \infty$, there is a constant $\gamma=\gamma(r)$, such that the growth condition

$$
\|F(t, u)\| \leq \gamma\|u\|, \quad(u \in \Omega(r), t \geq 0) .
$$

Remark 3. If, for some $p>1$, there is a constant $\gamma(p)>0$ such that

$$
\|F(t, u)\| \leq \gamma(p)\|u\|^{p}, \quad\left(u \in R^{n} ; t \geq 0\right)
$$

then for any fixed $r>0$, condition (4) holds with $\gamma=\gamma(r)=$ $\gamma(p) r^{p-1}$.

The logarithmic norm of a square matrix $B$ is defined by

$$
\mu(B)=\lim _{\varepsilon \rightarrow 0^{+}} \frac{\|I+\varepsilon B\|-1}{\varepsilon} .
$$

This logarithmic norm is often used as measure of stability and asymptotic decay in analytic and numerical studies concerning to ordinary differential equations (see [14, 20]). Its dependence upon the vector norm and matrix norms under consideration is clear. 
Remark 4. Different norms in $R^{n \times n}$ give rise to different logarithmic norms. However, independently of the considered norm, a logarithmic norm always has the following properties (see [7]).

Lemma 5. Let $B$ be a square matrix. Then

(i) $\mu(B)=\lim _{\varepsilon \rightarrow 0^{+}}\left(\left(\ln \left\|e^{\varepsilon B}\right\|\right) / \varepsilon\right), \quad \mu(B)=\min \left\{\lambda:\left\|e^{B t}\right\| \leq\right.$ $\left.e^{\lambda t}, t \geq 0\right\}$, and $\left\|e^{B t}\right\| \leq 1$ if and only if $\mu(B) \leq 0$;

(ii) for any norm, we have

$$
-\|B\| \leq-\mu(-B) \leq \operatorname{Re} \sigma(B) \leq \mu(B) \leq\|B\| ;
$$

(iii) $B$ is a stable matrix if and only if there exists a logarithmic norm $\mu$ such that $\mu(B)<0$.

For the 1-norm $\|x\|_{1}=\sum_{i=1}^{n}\left|x_{i}\right|$, the induced matrix measure $\mu_{1}$ is given by

$$
\mu_{1}(B)=\max _{j}\left\{b_{j j}+\sum_{i \neq j}^{n}\left|b_{i j}\right|\right\} .
$$

For the $\infty$-norm $\|x\|_{\infty}=\max _{1 \leq i \leq n}\left|x_{i}\right|$, the induced logarithmic norm is given by

$$
\mu_{\infty}(B)=\max _{i}\left\{b_{i i}+\sum_{j \neq i}\left|b_{i j}\right|\right\} .
$$

Remark 6. Although logarithmic norm is only defined for constant fixed matrices, it can be applied to any matrix, either time-invariant or time-varying. Thus, logarithmic norm technique can be used to study the stability of linear time-varying systems (Coppel $[7,15]$ ).

\section{Main Results}

The results described here are based upon the following Coppel's inequality ([14]):

$$
\left\|e^{B t}\right\| \leq e^{\mu(B) t}, \quad \forall t \geq 0
$$

where $\mu$ is a logarithmic norm of a square matrix $B$.

To establish our main results we make two basic assumptions on the coefficients of system (1):

$\left(\mathrm{H}_{1}\right)$ There is a positive real number $q$ such that

$$
\|A(t)-A(s)\| \leq q|t-s|, \quad \forall t, s \geq 0 .
$$

$\left(\mathrm{H}_{2}\right)$ For any logarithmic norm $\mu$, the matrix $A(t)$ satisfies

$$
\rho:=-\sup _{t \geq 0} \mu(A(t))>0 .
$$

Remark 7. Condition (12) works on matrices $A(t)$, for every fixed $t \geq 0$.

Theorem 8. Suppose that conditions $\left(H_{1}\right),\left(H_{2}\right)$, and (4) are satisfied. In addition, let

$$
G(A(\cdot), F)=\frac{q}{\rho^{2}}+\frac{\gamma}{\rho}<1
$$

Then the zero solution of (1) is exponentially stable with respect to a ball $\Omega(\lambda)$, with $\lambda=r(1-G(A(\cdot), F))$, provided that

$$
\|x(0)\|<r(1-G(A(\cdot), F)) .
$$

Proof. Let us take an initial value $x(0) \in \Omega(\lambda)$, satisfying inequality (14).

Rewrite system (1) in the form

$$
\frac{d x}{d t}=A(\tau) x(t)+[A(t)-A(\tau)] x(t)+F(t, x(t)),
$$

regarding an arbitrary $\tau \geq 0$ as fixed. If $x(t)$ is a solution of system (15), then

$$
\begin{gathered}
x(t)=\exp [A(\tau) t] x(0)+\int_{0}^{t} \exp [A(\tau)(t-s)] \\
\cdot\{(A(s)-A(\tau)) x(s)+F(s, x(s))\} d s .
\end{gathered}
$$

There are two cases to consider: $r=\infty$ and $r<\infty$, respectively. First, assume that (4) is valid with $r=\infty$; then taking norms of both sides and using conditions $\left(\mathrm{H}_{1}\right),(4)$, and (12), we obtain the relation

$$
\begin{aligned}
\|x(t)\| \leq & e^{\mu(A(\tau)) t}\|x(0)\| \\
& +\int_{0}^{t} e^{\mu(A(\tau))(t-s)}[q|s-\tau|+\gamma]\|x(s)\| d s .
\end{aligned}
$$

Taking $t=\tau$, we get

$$
\begin{aligned}
\|x(\tau)\| \leq & e^{\mu(A(\tau)) \tau}\|x(0)\| \\
& +\int_{0}^{\tau} e^{\mu(A(\tau))(\tau-s)}[q|s-\tau|+\gamma]\|x(s)\| d s .
\end{aligned}
$$

Hence, for any finite $T$, with $0 \leq \tau \leq T$, we have

$$
\begin{aligned}
\sup _{0 \leq \tau \leq T} & \|x(\tau)\| \\
\leq & \sup _{0 \leq \tau \leq T}\left(e^{\mu(A(\tau)) \tau}\right)\|x(0)\| \\
& +q \sup _{0 \leq \tau \leq T}\|x(\tau)\| \int_{0}^{T} e^{\mu(A(T))(T-s)}(T-s) d s \\
& +\gamma \sup _{0 \leq \tau \leq T}\|x(\tau)\| \int_{0}^{T} e^{\mu(A(T))(T-s)} d s .
\end{aligned}
$$

The last inequalities yield

$$
\begin{aligned}
\sup _{t \leq T}\|x(t)\| \\
\leq \sup _{t \geq 0}\left(e^{-\rho t}\right)\|x(0)\| \\
\quad+q \sup _{s \leq T}\|x(s)\| \int_{0}^{T} e^{\mu(A(T))(T-s)}(T-s) d s \\
\quad+\gamma \sup _{0 \leq s \leq T}\|x(s)\| \int_{0}^{T} e^{\mu(A(T))(T-s)} d s .
\end{aligned}
$$


But

$$
\begin{aligned}
& \int_{0}^{t} e^{\mu(A(t))(t-s)}(t-s) d s \leq \int_{0}^{t} e^{-\rho(t-s)}(t-s) d s \\
& \quad \leq \int_{0}^{\infty} e^{-\rho z} z d z .
\end{aligned}
$$

Hence

$$
\int_{0}^{t} e^{\mu(A(t))(t-s)}(t-s) d s \leq \frac{1}{\rho^{2}} .
$$

Proceeding in a similar way, we have

$$
\int_{0}^{t} e^{\mu(A(t))(t-s)} d s \leq \int_{0}^{t} e^{-\rho(t-s)} d s \leq \int_{0}^{\infty} e^{-\rho z} d z=\frac{1}{\rho} .
$$

By (17), (22), and (23), we get

$$
\sup _{t \leq T}\|x(t)\| \leq\|x(0)\|+\sup _{s \leq T}\|x(s)\|\left[\frac{q}{\rho^{2}}+\frac{\gamma}{\rho}\right] .
$$

But

$$
\frac{q}{\rho^{2}}+\frac{\gamma}{\rho}=G(A(\cdot), F)
$$

Consequently,

$$
\sup _{t \leq T}\|x(t)\| \leq[1-G(A(\cdot), F)]^{-1}\|x(0)\| .
$$

The right-hand side of (26) does not depend on $T$. Hence, condition $\|x(0)\|<\lambda$, with $\lambda=r(1-G(A(\cdot), F))$, ensures this bound for all $T \geq 0$. Thus, it follows that

$$
\sup _{t \geq 0}\|x(t)\| \leq[1-G(A(\cdot), F)]^{-1}\|x(0)\| .
$$

Bound (27) proves the Lyapunov stability with respect to the ball $\Omega(\lambda)$, with $\lambda=r(1-G(A(\cdot), F))$.

Now, let $r<\infty$. Then by a simple application of the Urysohn's lemma [28, p.15]. and taking into account the relation

$$
[1-G(A(\cdot), F)]^{-1}\|x(0)\|<r
$$

we get the Lyapunov stability in this case.

To establish the exponential stability with respect to the ball $\Omega(\lambda)$ of the zero solution of system (1), let us define a new variable

$$
x_{\alpha}(t)=x(t) e^{\alpha t},
$$

with a small enough $\alpha>0$. Here $x(t)$ is a solution of (1). Substituting (29) in (1), we obtain

$$
\frac{d x_{\alpha}}{d t}=A_{\alpha}(t) x_{\alpha}(t)+F_{1}\left(t, x_{\alpha}(t)\right), \quad t \geq 0,
$$

where $A_{\alpha}(t)=\alpha I+A(t)$, and

$$
F_{1}(t, u)=e^{\alpha t} F\left(t, e^{-\alpha t} u\right) ; \quad(u \in \Omega(\lambda) ; t \geq 0) .
$$

The growth condition (4) yields

$$
\left\|F_{1}(t, u)\right\| \leq e^{\alpha t} F\left(t, e^{-\alpha t} u\right) ; \quad(u \in \Omega(\gamma), t \geq 0) .
$$

Applying our reasoning above to (30), we get according to inequality (27) that $x_{\alpha}(t)$ is a bounded function. Hence (29) yields the exponential stability.

Remarks 2. (a) Notice that this theorem is valid for an arbitrary logarithmic norm. For the specific case of $\mu_{1}$, Theorem 8 extends some results given by $[3,10,25]$.

(b) Theorem 8 asserts that any initial condition $x_{0} \in \Omega(\lambda)$, satisfying the condition

$$
\|x(0)\|<r(1-G(A(\cdot), F)),
$$

belongs to the region of attraction.

(c) If $G(A(\cdot), F)=0$, then any solution of (1) with initial value

$$
x(0) \in\{u \in \Omega(\lambda):\|u\| \leq r\}
$$

is bounded.

Example 9. Let us illustrate the obtained results by the following example:

Consider the nonlinear system in $R^{2}$ given by

$$
\begin{aligned}
\frac{d x_{1}}{d t}= & -\left[a_{1}+d_{1}(t)\right] x_{1}(t)+d_{2}(t) x_{2}(t) \\
& +\gamma_{1} x_{1}(t) e^{-\alpha_{1} x_{1}(t)} \\
\frac{d x_{2}}{d t}= & -\left[a_{2}+d_{2}(t)\right] x_{2}(t)+d_{1}(t) x_{1}(t) \\
& +\gamma_{2} x_{2}(t) e^{-\alpha_{2} x_{2}(t)}
\end{aligned}
$$

where $a_{i}(i=1,2)$ and $\alpha_{i}>0$ and $\gamma_{i}>0(i=1,2)$ are constants. $d_{i}(t)(i=1,2)$ are bounded nonnegative functions.

Rewrite system (35) in the vector form of (1):

$$
\frac{d x}{d t}=A(t) x(t)+f(x(t))
$$

where $A(t)=\left[\begin{array}{cc}-\left(a_{1}+d_{1}(t)\right) & d_{2}(t) \\ d_{1}(t) & -\left(a_{2}+d_{2}(t)\right)\end{array}\right], x(t)=\left(x_{1}(t), x_{2}(t)\right)^{T}$, $f(x(t))=\left(f_{1}, f_{2}\right)^{T}$, and

$$
f_{i}\left(x_{i}(t)\right)=\gamma_{i} x_{i}(t) e^{-\alpha_{i} x_{i}(t)}, \quad i=1,2 .
$$

Define

$$
\begin{aligned}
& q=\max \left\{2 q_{1}, 2 q_{2}\right\}, \\
& \qquad q_{i}>0 \text { are constants for } i=1,2 .
\end{aligned}
$$

Theorem 10. Assume that there exist constants $q_{i}>0(i=$ $1,2)$ such that

$$
\left|d_{i}(t)-d_{i}(s)\right| \leq q_{i}|t-s|, \quad \forall t, s \geq 0 \quad(i=1,2) .
$$


In addition, let

$$
\begin{aligned}
\min \left\{a_{1}, a_{2}\right\} & >\max \left\{\gamma_{1}, \gamma_{2}\right\}, \\
\frac{q}{\left(\min \left\{a_{1}, a_{2}\right\}\right)^{2}}-\frac{\max \left\{\gamma_{1}, \gamma_{2}\right\}}{\min \left\{a_{1}, a_{2}\right\}}<1 . &
\end{aligned}
$$

Then the zero solution of system (36) is exponentially stable with respect to a ball $\Omega\left(\lambda_{0}\right)$, with $\lambda_{0}=r\left(1-S_{0}\right)$, provided that $\|x(0)\|<r\left(1-S_{0}\right)$, where

$$
S_{0}=\frac{q}{\left(\min \left\{a_{1}, a_{2}\right\}\right)^{2}}-\frac{\max \left\{\gamma_{1}, \gamma_{2}\right\}}{\min \left\{a_{1}, a_{2}\right\}} .
$$

Proof. Define the matrix norm by $\|B\|=\max _{1 \leq j \leq 2} \sum_{i=1}^{2}\left|b_{i j}\right|$; then

$$
\mu(B)=\max _{1 \leq j \leq 2}\left\{b_{j j}+\sum_{k=1, k \neq j}^{2}\left|b_{k j}\right|\right\} .
$$

Hence, for small $\varepsilon>0$, we have

$$
\begin{aligned}
\| I & +\varepsilon A(t) \| \\
& =\left\|\left(\begin{array}{cc}
1-\varepsilon\left(a_{1}+d_{1}(t)\right) & \varepsilon d_{2}(t) \\
\varepsilon d_{1}(t) & 1-\varepsilon\left(a_{2}+d_{2}(t)\right)
\end{array}\right)\right\| \\
& =\max \left\{1-\varepsilon a_{1}, 1-\varepsilon a_{2}\right\}=1-\varepsilon \min \left\{a_{1}, a_{2}\right\} .
\end{aligned}
$$

Thus,

$$
\mu(A(t))=-\min \left\{a_{1}, a_{2}\right\} ; t \geq 0 .
$$

To prove the exponential stability of the zero solution of (36), it suffices that condition (4) of Theorem 8 holds for any vector $v=\left(v_{1}, v_{2}\right)^{T} \in \Omega(r)$, and the coefficients $A(t), t \geq 0$ are slowly varying; that is, there exists $q>0$ such that

$$
\|A(t)-A(s)\| \leq q|t-s|, \quad \forall t, s \geq 0 .
$$

By (39), simple calculations show that

$$
\|A(t)-A(s)\| \leq \max \left\{2 q_{1}, 2 q_{2}\right\}|t-s|, \quad \forall t, s \geq 0 .
$$

On the other hand, by (37), it follows that

$$
\begin{aligned}
&\|f(x)\| \leq \\
& \leq\left\|\operatorname{diag}\left(\gamma_{1}, \gamma_{2}\right)\left(\begin{array}{l}
x_{1} e^{-\alpha_{1} x_{1}} \\
x_{2} e^{-\alpha_{2} x_{2}}
\end{array}\right)\right\| \operatorname{diag}\left(\gamma_{1}, \gamma_{2}\right)\|\| x\left\|=\max \left\{\gamma_{1}, \gamma_{2}\right\}\right\| x \|, \\
& x \in \Omega(r) .
\end{aligned}
$$

Hence, by Theorem 8 the zero solution of system (35) is exponentially stable.

\section{Pseudo-Linear Systems}

Pseudo-linear systems are an important class of nonlinear systems. Theorem 8 will be a fundamental theorem to establish the stability and robustness of this kind of differential systems.
Consider in $R^{n}$ the equation

$$
\frac{d x}{d t}=B(x(t), t) x(t), \quad t \geq 0,
$$

where $B(z, t)$ continuously depends on $z \in \Omega(r)$ and $t \geq 0$. Everywhere below, it is assumed that $B(z, t)$ are bounded linear operators in $R^{n}$ for each $z \in \Omega(r), t \geq 0$. The existence of solutions of (48) is assumed.

It is assumed that there are constants $\eta(r)$ and $v(r)$ such that

$$
\begin{gathered}
\left\|B\left(h_{1}, t\right)-B(h, t)\right\| \leq v(r)\left\|h_{1}-h\right\|, \\
\|B(h, t)-B(h, s)\| \leq \eta(r)\|t-s\|,
\end{gathered}
$$

for $h, h_{1} \in \Omega(r) ; t, s \geq 0$.

$$
M(r)=\sup _{h \in \Omega(r), t \geq 0}\|B(h, t) h\|,
$$

and $q_{0}(r)=v(r) M(r)+\eta(r)$.

Assume that, for any logarithmic norm $\mu$, the matrix $B(h(t), t)$ satisfies $\mu(B(h(t), t))<0$ for every fixed $t \geq 0$. Namely,

$$
\tilde{\rho}=-\sup _{h \in \Omega(r), s \geq 0} \mu(B(h, s))>0 .
$$

Remark 11. Here we will consider system (48) as a perturbation of a fixed operator $B\left(x_{0}, t_{0}\right)$. Thus, our results can be applied to robust stability; in fact, we can regard

$$
\frac{d x}{d t}=B\left(x_{0}, t_{0}\right) x(t), \quad t \geq 0
$$

as the nominal system and

$$
\frac{d x}{d t}=B(x, t) x
$$

as a system with state-dependent parametric perturbations.

Theorem 12. For a positive $r<\infty$, assume conditions (49)-(51) and

$$
\frac{q_{0}(r)}{\widetilde{\rho}^{2}}<1
$$

Then the zero solution of system (48) is exponentially stable with respect to a ball $\Omega(\lambda)$ with $\lambda=r\left(1-q_{0}(r) / \widetilde{\rho}^{2}\right)$, provided that

$$
\|x(0)\|<r\left(1-\frac{q_{0}(r)}{\widetilde{\rho}^{2}}\right) .
$$

Proof. Let us introduce the linear equation

$$
\frac{d y_{h}}{d t}=B(h(t), t) y_{h}, \quad t \geq 0
$$

where $h:[0, \infty) \rightarrow R^{n}$ is a differentiable function. If $h(t)=$ $x(t)$ is a solution of (48), then (48) and (56) coincide. Further, 
the continuous dependence of solution on initial data implies that, under condition $\|x(0)\|<r$, there is $t_{0}$, such that

$$
x(t) \in \Omega(r) \quad \text { for } 0 \leq t \leq t_{0}
$$

for a solution $x(t)$ of (48). If we put $A(t)=B(h(t), t)$ with some function $h(t)$, then (48) takes the form

$$
\frac{d x}{d t}=A(t) x
$$

Due to (49), we have

$$
\begin{aligned}
&\|B(h(t), t)-B(h(s), s)\| \\
& \leq\|B(h(t), t)-B(h(s), t)\| \\
& \quad+\|B(h(s), t)-B(h(s), s)\| \\
& \leq v(r)\|h(t)-h(s)\|+\eta(r)|t-s| ; \quad\left(t, s \leq t_{0}\right) .
\end{aligned}
$$

Hence,

$$
\begin{aligned}
& \|B(x(t), t)-B(x(s), s)\| \\
& \quad \leq v(r)\|x(t)-x(s)\|+\eta(r)|t-s| \\
& \quad \leq v(r) \sup _{t \leq t_{0}}\left\|\frac{d x}{d t}\right\||t-s|+\eta(r)|t-s| ;
\end{aligned}
$$

$$
\left(t, s \leq t_{0}\right)
$$

But according to (48),

$$
\left\|\frac{d x}{d t}\right\| \leq M(r), \quad\left(t \leq t_{0}\right) .
$$

Thus,

$$
\|B(x(t), t)-B(x(s), s)\| \leq q_{0}(r)|t-s| ;
$$

$$
\left(t, s \leq t_{0}\right) \text {. }
$$

Applying Theorem 8, with $A(t)=B(x(t), t)$ to (48), we have the bound

$$
\sup _{t \leq t_{0}}\|x(t)\| \leq\left(1-\frac{q_{0}(r)}{\widetilde{\rho}^{2}}\right)\|x(0)\|, \quad t \leq t_{0} .
$$

But condition (55) allows us to extend this bound to all $t \geq 0$. Hence, estimate (63) is proved for all $t \geq 0$. It yields the Lyapunov stability. Following the lines of Theorem 8 , the exponential stability is proven.

Remark 13. Theorem 12 remains true if the linear system

$$
\frac{d x}{d t}=B(0, t) x, \quad t \geq 0
$$

satisfies (50). In this case condition (51) takes the form $\hat{\rho}=$ $-\sup _{t \geq 0} \mu(B(0, t))>0$, and (54) becomes $\eta(r) / \widehat{\rho}^{2}<1$.

\section{Application}

Consider the following approximation to (1):

$$
\frac{d x}{d t}=A(t) x, \quad x\left(t_{0}\right)=x_{0} .
$$

Denote by $U\left(t, t_{0}\right)$ the fundamental matrix of this system. We assume that

$$
\begin{gathered}
\sup _{t \geq t_{0}}\left\|U\left(t, t_{0}\right)\right\| \leq m_{1}\left(t_{0}\right), \\
\sup _{t \geq t_{0}}\left\|U^{-1}\left(t, t_{0}\right)\right\| \leq m_{2}\left(t_{0}\right) .
\end{gathered}
$$

Make the change of variables $x(t)=U\left(t, t_{0}\right) y(t)$ in (1). Hence, (1) reduces to the form

$$
\frac{d y}{d t}=U^{-1}\left(t, t_{0}\right) F\left(t, U\left(t, t_{0}\right) y(t)\right)
$$

$$
y\left(t_{0}\right)=y_{0}=x_{0} .
$$

Assume that the right-hand side of (67) reduces to the form

$$
U^{-1}\left(t, t_{0}\right) F\left(t, U\left(t, t_{0}\right) y(t)\right)=\widetilde{B}(y, t) y .
$$

We additionally assume that $\widetilde{B}(y, t)$ satisfies conditions (49)(50) applied to (67); that is,

$$
\begin{aligned}
\widetilde{M}(r) & =\sup _{h \in \Omega(r), t \geq t_{0}}\|\widetilde{B}(h, t) h\|, \\
\widetilde{q}_{0}(r) & =v(r) \widetilde{M}(r)+\widetilde{\eta}(r), \\
\widehat{\rho} & =-\sup _{h \in \Omega(r), s \geq 0} \mu(\widetilde{B}(h, s))>0 .
\end{aligned}
$$

Theorem 14. For a positive $r<\infty$, assume conditions (49) and (69) hold. In addition, assume that

$$
\widetilde{q}_{0}(r)\left(\widehat{\rho}^{2}\right)^{-1}<1 .
$$

Then the zero solution of system (65) is exponentially stable with respect to a ball $\Omega(\widehat{\lambda})$, with $\hat{\lambda}=r\left(1-\tilde{q}_{0}(r)\left(\hat{\rho}^{2}\right)^{-1}\right)$, provided that

$$
\|x(0)\|<r\left(1-\tilde{q}_{0}(r)\left(\hat{\rho}^{2}\right)^{-1}\right) .
$$

Proof. The proof follows directly from Theorem 12.

\section{Conclusions}

New conditions for the exponential stability for nonlinear finite-dimensional differential systems as well as a class of finite-dimensional pseudo-linear systems are derived. We establish the robustness of the exponential stability, in the sense that the exponential stability for a given pseudo-linear equation persists under sufficiently small perturbations. It is shown for finite-dimensional systems that the local frozen time analysis is justifiable for the systems with Hölder-like 
continuity which is broader than the class of slow-varying systems. The proofs are carried out using the semigroup theory combined with the freezing method and the logarithmic technique. That is, the equation is represented as a perturbation about a fixed value of the operator and then applying norm estimates for operator-valued functions the results follow. We have presented an example which shows how this approach bring out different aspects of the stability problem of pseudo-linear equations. Finally, an application of the exponential stability results for pseudo-linear differential systems is applied to an approximation to (1).

\section{Conflicts of Interest}

The author declares that there are no conflicts of interest regarding the publication of this paper.

\section{Acknowledgments}

This research was supported by Direccion de Investigacion under Grant NU 06/16 (Chile).

\section{References}

[1] L. Barreira and C. Valls, Stability of Nonautonomous Differential Equations, vol. 1926 of Lecture Notes in Mathematics, Springer, Berlin, Germany, 2008.

[2] W. M. Haddad, V. Chellaboina, and Q. Hui, Nonnegative and Compartmental Dynamical Systems, Princeton University Press, Princeton, NJ, USA, 2010

[3] X. Liu and J. Lam, "Relationships between asymptotic stability and exponential stability of positive delay systems," International Journal of General Systems, vol. 42, no. 2, pp. 224-238, 2013.

[4] J. Peuteman and D. Aeyels, "Averaging results and the study of uniform asymptotic stability of homogeneous differential equations that are not fast time-varying," SIAM Journal on Control and Optimization, vol. 37, no. 4, pp. 997-1010, 1999.

[5] V. Solo, "On the stability of slowly time-varying linear systems," Mathematics of Control, Signals, and Systems, vol. 7, no. 4, pp. 331-350, 1994.

[6] P. van den Driessche and X. Zou, "Global attractivity in delayed Hopfield neural network models," SIAM Journal on Applied Mathematics, vol. 58, no. 6, pp. 1878-1890, 1998.

[7] M. Vidyasagar, Nonlinear Systems Analysis, Prentice Hall, New York, NY, USA, 1993.

[8] S. P. Banks, A. Moser, and D. McCaffrey, "Robust exponential stability of evolution equations," Polish Academy of Sciences, vol. 4, no. 3-4(40), pp. 261-279, 1995.

[9] J. Cao and L. Wang, "Exponential stability and periodic oscillatory solution in BAM networks with delays," IEEE Transactions on Neural Networks, vol. 13, no. 2, pp. 457-463, 2002.

[10] K. S. Hong, J. W. Wu, and K.-I. Lee, "New conditions for the exponential stability of evolution equations," IEEE Transactions on Automatic Control, vol. 39, no. 7, pp. 1432-1436, 1994.

[11] P. H. Ngoc, "On exponential stability of nonlinear differential systems with time-varying delay," Applied Mathematics Letters, vol. 25, no. 9, pp. 1208-1213, 2012.
[12] A. Zevin and M. Pinsky, "Exponential stability and solution bounds for systems with bounded nonlinearities," IEEE Transactions on Automatic Control, vol. 48, no. 10, pp. 1799-1804, 2003.

[13] Y. B. Wang and Z. J. Han, "A stability property of nonlinear systems with slowly varying inputs," Control Theory \& Applications, vol. 16, no. 2, pp. 158-162, 1999.

[14] W. A. Coppel, Stability and Asymptotic Behavior of Differential Equations, D. C. Heath, Boston, Mass, USA, 1965.

[15] W. A. Coppel, Dichotomies in Stability Theory, vol. 629 of Lecture Notes in Mathematics, Vol. 629, Springer, Berlin, Germany, 1978.

[16] J. K. Hale and S. M. Verduyn Lunel, Introduction to FunctionalDifferential Equations, vol. 99 of Applied Mathematical Sciences, Springer, New York, NY, USA, 1993.

[17] B. D. O. Anderson, A. Ilchmann, and F. R. Wirth, "Stabilizability of linear time-varying systems," Systems and Control Letters, vol. 62, no. 9, pp. 747-755, 2013.

[18] R. E. Vinograd, "An improved estimate in the method of freezing," Proceedings of the American Mathematical Society, vol. 89, no. 1, pp. 125-129, 1983.

[19] M. I. Gil and R. Medina, "The freezing method for linear difference equations," Journal of Difference Equations and Applications, vol. 8, no. 5, pp. 485-494, 2002.

[20] G. Dahlquist, "Stability and error bounds in the numerical integration of ordinary differential equations," Kungliga Tekniska Högskolan, Stockholm, vol. 30, p. 87, 1959.

[21] S. M. Lozinskii, "Error estimates for the numerical integration of ordinary differential equations," Izvestiya Vysshikh Uchebnykh Zavedenii. Matematika, vol. 6, no. 5, pp. 52-90, 1958 (Russian).

[22] J. M. Ferreira and I. Györi, "Oscillatory behavior in linear retarded functional differential equations," Journal of Mathematical Analysis and Applications, vol. 128, no. 2, pp. 332-346, 1987.

[23] A. I. Dvirnyi and V. I. Slyn'ko, "Stability of solutions to impulsive differential equations in critical cases," Siberian Mathematical Journal, vol. 52, no. 1, pp. 54-62, 2011.

[24] A. I. Dvirnyi and V. I. Slyn'ko, "Global stability of solutions of nonstationary monotone differential equations with impulse action in a pseudolinear form," Nonlinear Oscillations, vol. 14, no. 2, pp. 193-210, 2011.

[25] A. A. Martynyuk, "Novel bounds for solutions of nonlinear differential equations," Applied Mathematics, vol. 6, no. 1, pp. 182-194, 2015.

[26] K. Furuta and S. B. Kim, "Pole assignment in a specified disk," IEEE Transactions on Automatic Control, vol. 32, no. 5, pp. 423427, 1987.

[27] F.-H. Hsiao, J.-D. Hwang, and S.-P. Pan, "D-stability analysis for discrete uncertain time-delay systems," Applied Mathematics Letters, vol. 11, no. 2, pp. 109-114, 1998.

[28] N. Dunford and J. T. Schwarz, Linear Operators, Part I, Wiley Interscience, New York, NY, USA, 1966. 


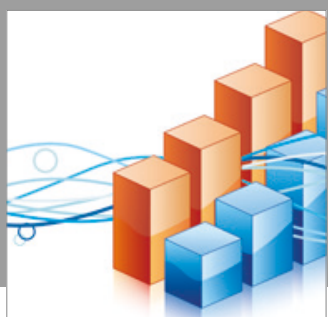

Advances in

Operations Research

vatersals

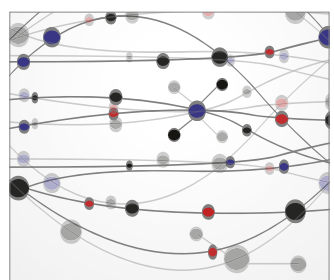

\section{The Scientific} World Journal
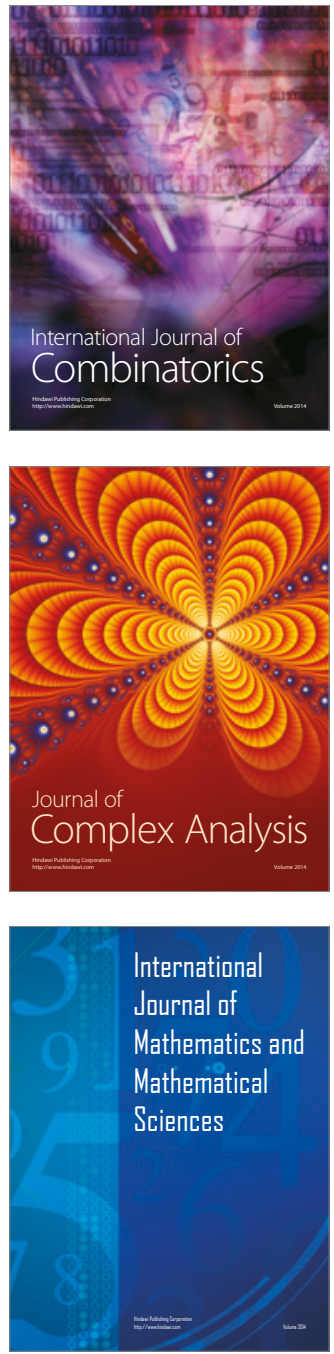
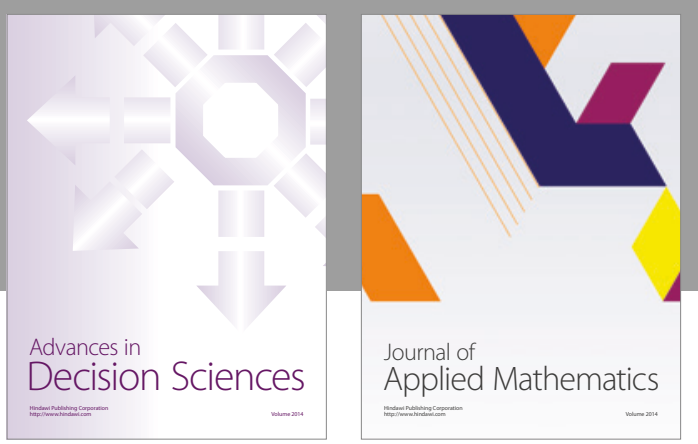

Algebra

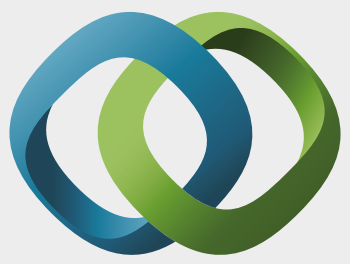

\section{Hindawi}

Submit your manuscripts at

https://www.hindawi.com
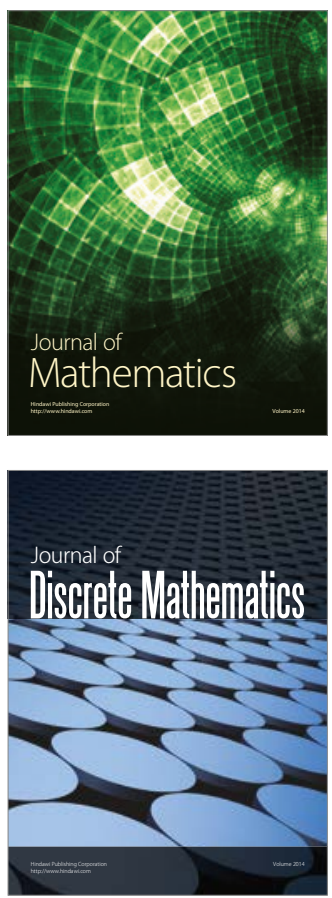

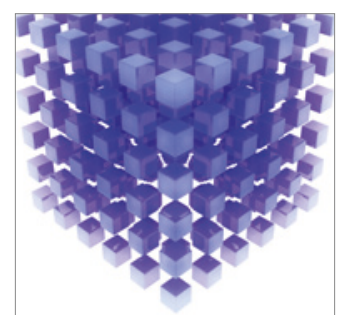

Mathematical Problems in Engineering
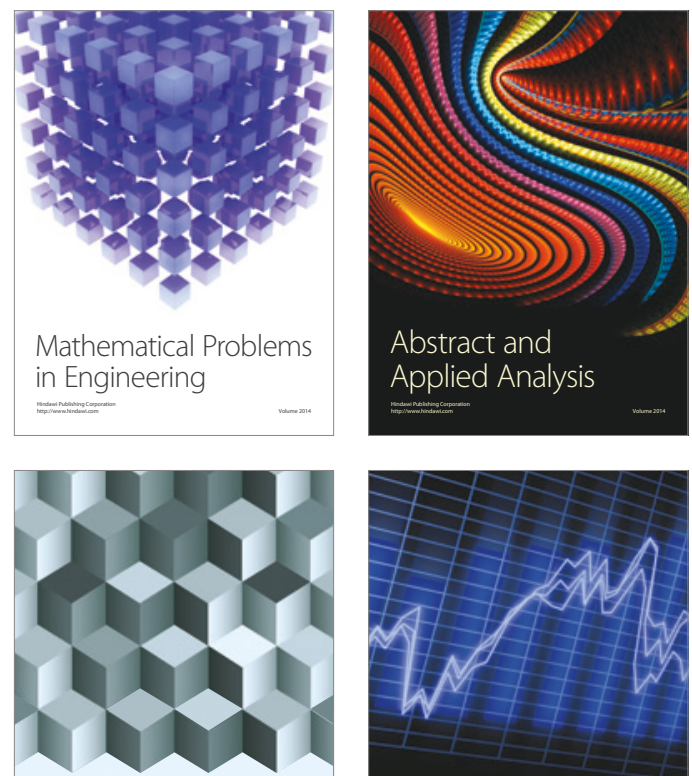

Journal of

Function Spaces

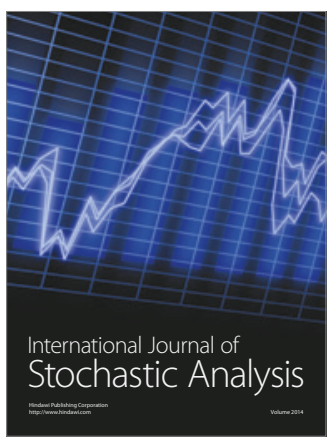

Probability and Statistics
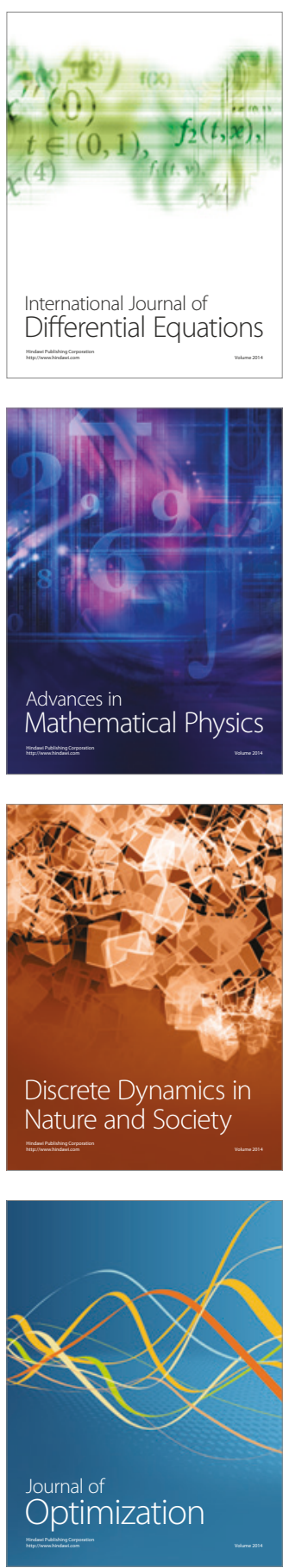